\title{
Constructing thin subgroups commensurable with the figure-eight knot group
}

\author{
SAMUEL BALLAS \\ DARREN D LONG
}

\begin{abstract}
We find infinitely many lattices in $\operatorname{SL}(4, \mathbf{R})$, each of which contains thin subgroups commensurable with the figure-eight knot group.
\end{abstract}

$57 \mathrm{M} 60$

\section{Introduction}

Let $\Gamma$ be a lattice in a semisimple Lie group $G$. Then following Sarnak [22], one says that a subgroup $\Delta$ of $\Gamma$ is thin if $\Delta$ has infinite index in $\Gamma$, and is Zariski dense in $G$. Since it is rather easy to exhibit Zariski dense subgroups of lattices that are free products, the case of most interest is that the thin group $\Delta$ is finitely generated and does not decompose as a free product.

In this note, we shall exhibit subgroups of the fundamental group of the figure-eight knot as subgroups of infinitely many incommensurable lattices. A precise statement will be given shortly.

We begin by briefly describing where the lattices in question arise. They were constructed by Morris [18, Theorem 6.55] by a rather general construction which involves $L$, a real quadratic extension of $\mathbf{Q}$ and $D$, a central simple division algebra of degree $d$ over $L$. However in our situation we may assume that $D=L$, and we state only this special case.

In this paper, the field $L$ is a real quadratic extension of $\mathbf{Q}$ and if $A \in \operatorname{SL}(4, L)$, we denote by $A^{*}$ the matrix obtained by taking the transpose of the matrix obtained from $A$ by applying $\tau$ (the nontrivial Galois automorphism) to all its entries. Then one has:

Theorem 1.1 Suppose that $L$ is a real quadratic extension of $\mathbf{Q}$, with Galois automorphism $\tau$. Suppose that $b_{1}, \ldots, b_{4}$ are nonzero elements of $\mathbf{Z}$. Set $J=\operatorname{diag}\left(b_{1}, \ldots, b_{4}\right)$. Then the group

$$
\mathrm{SU}\left(J, \mathcal{O}_{L}, \tau\right)=\left\{A \in \mathrm{SL}\left(4, \mathcal{O}_{L}\right) \mid A^{*} J A=J\right\}
$$

is a lattice in $\operatorname{SL}(4, \mathbf{R})$. 
We also note from Morris [18, Proposition 6.55] that, in the case being considered here (when $D=L$ ), the corresponding forms will represent zero nontrivially, and so the lattices produced are nonuniform.

We shall show:

Theorem 1.2 Let $\Gamma$ denote the fundamental group of the figure-eight knot and $L_{d}=$ $\mathbf{Q}(\sqrt{d})$ for $d$ a positive, square-free integer. Then for every such $d$, there is a subgroup of finite index in $\Gamma, H_{d}$, and a faithful, Zariski dense representation into a lattice:

$$
r_{d}: H_{d} \longrightarrow \operatorname{SU}\left(J_{d}, \mathcal{O}_{L_{d}}, \tau_{d}\right)
$$

The power of this statement lies in the fact that $r_{d}$ is faithful and Zariksi dense; much of the work of the paper is devoted to this aspect. Once one knows this, it follows immediately that the image group $r_{d}\left(H_{d}\right)$ has infinite index in the lattice $\operatorname{SU}\left(J_{d}, \mathcal{O}_{L_{d}}, \tau_{d}\right)$, since deep results of Margulis (see Morris [18, Chapter 13]) imply that such lattices do not have subgroups which admit homomorphisms onto $\mathbf{Z}$. Moreover, subgroups of finite index in $\Gamma$ are freely indecomposable, so that it follows from Theorem 1.2 that the image is thin.

This paper is organized as follows. The starting point is a pair of representations of $\Gamma$ constructed independently by Ballas [3] (which we denote $\phi_{t}$ ) and Thistlethwaite (private communication) (which we denote $\rho_{v}$ ). These representations are conjugate when $v=2 t$ and while one could work with just one of them, each presents sufficiently interesting features that it seems worthwhile to include both. Explicit generators for both representations are included in the appendix. We begin in Section 2 with the algebraic considerations needed to construct the representations $r_{d}$ of Theorem 1.2 from the Ballas-Thistlethwaite representation. The main work here is proving that the representation has an integral character in an appropriate sense; the remaining ingredients in constructing $r_{d}$ are fairly standard once this has been proved.

The results of Section 3 and Section 4 are geometric and lie deeper. Specifically, Section 3 is devoted to the proof that the representations $r_{d}$ are faithful using results from real projective geometry.

In Section 4 we show that, with the exception of the representation corresponding to the complete hyperbolic structure, $\phi_{t}$ has Zariski dense image in $\operatorname{SL}(4, \mathbf{R})$.

\section{Constructing $r_{d}$}

This section is devoted to outlining the computations necessary to exhibit the representations $r_{d}$ of Theorem 1.2. With a view to a point that arises in the sequel (see Theorem 3.2), we begin by proving the following: 
Theorem 2.1 The representations $\rho_{v}$ are absolutely irreducible for real $v>0$.

Proof If there is an invariant subspace of dimension one, then any commutator must have eigenvalue $=1$. One can check that the commutator $\left[x^{2}, y\right]$ has such an eigenvalue only when $v$ is a root of $v^{2}+v+1$. The same considerations applied to the transpose representation show there can be no invariant subspace of $\rho_{v}$ of dimension three.

The case of a two-dimensional invariant subspace is more subtle. One computes that the characteristic polynomial of the longitude is $(-Q+v)^{3}\left(-1+Q v^{3}\right) / v^{3}$, so that if there were such an invariant subspace, either $\rho_{v}(\lambda)$ or $\rho_{v}^{T}(\lambda)$ would have the $1 / v^{3}$ eigenvalue appearing in that subspace. It follows that the orbit of that eigenvector would be two-dimensional. However, one can compute that this happens for neither of these representations.

Our next claim concerns the traces of the $\rho_{v}$.

Lemma 2.2 The representation of the figure-eight knot given by $\rho_{v}$ has traces lying in $\mathbf{Z}[v, 1 / v]$.

Proof This involves some computation, as it must. We indicate the mathematics behind the idea, with a sample of its implementation in [6].

Regarding $v$ as a real transcendental, using Burnside's theorem [14, page 648, Corollary 3.4], or by inspection, one can find elements $g_{1}, \ldots, g_{16} \in \Gamma$ which are a basis for the vector space of $4 \times 4$ matrices $M(4, \mathbf{R})$; we always choose $g_{1}$ to be the identity matrix, which we denote by $I$.

Let $g_{1}^{*}, \ldots, g_{16}^{*}$ be the dual basis with respect to trace, ie $\operatorname{tr}\left(g_{i} \cdot g_{j}^{*}\right)=\delta_{i j}$. One can use the action of the figure-eight knot group on this dual basis by left multiplication to obtain a 16-dimensional representation of the group, ie if $\gamma \in \Gamma$, then its action is defined by

$$
\gamma \cdot g_{i}^{*}=\sum_{j} \alpha_{i j}(\gamma) g_{j}^{*}
$$

Taking traces in this equation, we get

$$
\operatorname{tr}\left(\gamma \cdot g_{i}^{*}\right)=\sum_{j} \alpha_{i j}(\gamma) \operatorname{tr}\left(g_{j}^{*}\right) .
$$

Notice that since we have chosen $g_{1}=I$, we have that $\operatorname{tr}\left(g_{j}^{*}\right)=\operatorname{tr}\left(g_{1} \cdot g_{j}^{*}\right)=\delta_{1 j}$, in particular these are all rational integers. Writing $I=\sum \tau_{j} g_{j}^{*}$, notice that $\tau_{i}=\operatorname{tr}\left(g_{i}\right)$ by duality; one verifies that these traces only have denominators which are powers of $v$. 
Moreover, multiplying by $\gamma$ and taking traces, we have

$$
\operatorname{tr}(\gamma)=\sum_{j} \operatorname{tr}\left(g_{j}\right) \operatorname{tr}\left(\gamma \cdot g_{j}^{*}\right)=\sum_{j, k} \operatorname{tr}\left(g_{j}\right) \operatorname{tr}\left(g_{k}^{*}\right) \alpha_{j k}(\gamma)=\sum_{j} \operatorname{tr}\left(g_{j}\right) \alpha_{j 1}(\gamma) .
$$

The upshot of these two computations is the following: If with some choice of basis, one computes that the denominators of the entries for the associated $16 \times 16$ regular representation, then this collection of denominators contains the denominators for the traces of the original collection of matrices of $\Gamma$.

Therefore, if one could find a basis for which this construction gave $\mathbf{Z}[v, 1 / v]$-matrices $\left(\alpha_{i j}(\gamma)\right)$, then this would prove the result claimed by the lemma. However, this appears to be hard. We bypass this difficulty by constructing two representations via two different choices of basis $\left\{g_{i}\right\}$. For the first choice one sees all the matrix entries have denominators $v,(-1+v)$ and $(1+v)$, for the second, one sees denominators $v,\left(1+3 v+v^{2}\right)$ and $\left(1+3 v+4 v^{2}\right)$. Since traces are not dependent on choice of basis, the denominators of the trace of the original representation of $\Gamma$ must lie in the intersection of these two sets, ie powers of $v$.

Corollary 2.3 If one specializes $v$ to be a unit in any number field, then the resulting representation has integral trace.

Since when one sets $v=2 t$ the representation $\rho_{v}$ is conjugate to the representation $\phi_{t}$ and this representation has entries lying in $\mathbf{Q}(t)=\mathbf{Q}(v / 2)$, we are now in a position to apply the following lemma with $k=\mathbf{Q}(v / 2)$.

Theorem 2.4 Suppose that $G<\mathrm{SL}(4, k)$ is a finitely generated group with the property that $\operatorname{tr}(\gamma) \in \mathcal{O}_{k}$ for every $\gamma \in G$. Then $G$ has a subgroup of finite index contained in $\operatorname{SL}\left(4, \mathcal{O}_{k}\right)$.

Proof Consider

$$
\mathcal{O} G=\left\{\sum a_{i} \gamma_{i} \mid a_{i} \in \mathcal{O}_{k}, \gamma_{i} \in G\right\},
$$

where the sums are finite. It is shown in [7, Proposition 2.2 and Corollary 2.3] that $\mathcal{O} G$ is an order of a central simple subalgebra $B \subset M(4, k)$ defined over $k$. Now while $\mathcal{O} G$ need not be an order in $M(4, k)$, it is known that it is contained in some maximal order $\mathcal{D}$ of $M(4, k)$ (see [20, page 131, Exercise 5] and [15, proof of Lemma 2.3]).

Now it is a standard fact that the groups of elements of norm 1 in orders contained in $M(4, k)$ are commensurable (since the intersection of two orders is an order and the unit groups of orders will be irreducible lattices in $\operatorname{SL}(4, \mathbf{R}) \times \operatorname{SL}(4, \mathbf{R})[18$, Chapter $15 \mathrm{I}])$. In particular, $\operatorname{SL}\left(4, \mathcal{O}_{k}\right)$ and $\mathcal{D}^{1}$ are commensurable. Let $\Delta=\operatorname{SL}\left(4, \mathcal{O}_{k}\right) \cap \mathcal{D}^{1}$, which has finite index in both groups. Then $G \leq \mathcal{D}^{1}$, so that $G \cap \Delta$ has finite index in $G$ and lies inside $\operatorname{SL}\left(4, \mathcal{O}_{k}\right)$ as required. 
What have achieved to this point is that when one specializes $v$ to be any unit of a number field $k$, there is a representation, namely $\phi_{v / 2}$, of a subgroup of finite index in $\Gamma$ whose image has entries lying in $\mathcal{O}_{k}$.

We now turn our attention to the unitary aspects required by Theorem 1.1. There is an obvious involution on the matrices in the image of $\rho_{v}$ given by transposing and mapping $v \rightarrow 1 / v$. We denote this operation by $A \rightarrow A^{*}$. A routine computation on the generators reveals:

Lemma 2.5 There is a matrix $Q_{v}$ with $\operatorname{det}\left(Q_{v}\right) \neq 0$ for which

$$
A^{*} \cdot Q_{v} \cdot A=Q_{v}
$$

for all $A \in \phi_{v / 2}(\Gamma)$.

Since it's required by the result of Theorem 1.1, for the rest of this paper we assume $k=\mathbf{Q}(\sqrt{d})$ is a real quadratic number field equipped with involution $\tau_{d}$. Specializing $v$ to be a unit of this field with the property that $\tau_{d}(v)=1 / v$ (eg one can take the square of a random unit of $\mathbf{Q}(\sqrt{d})$; we call these the positive units), we see that Lemma 2.5 and Theorem 2.4 taken together prove:

Theorem 2.6 For each positive square free integer $d$, and a positive unit $u$ in the ring of integers of $\mathbf{Q}(\sqrt{d})$, there is a representation of a subgroup of finite index $H_{d, u}$ in $\Gamma$ :

$$
r_{d, u}: H_{d, u} \longrightarrow \mathrm{SU}\left(Q_{v}, \mathcal{O}_{k}, \tau_{d}\right)
$$

There is one final consideration that must be addressed, namely the form described by Witte is diagonal and $Q_{v}$ is not. However this concern is addressed as follows. It can easily be shown by using the Gram-Schmidt process for example, that there is a change of basis matrix $M_{d} \in \operatorname{GL}(\mathbf{Q}(\sqrt{d}))$ for which $M_{d}^{*} \cdot Q_{v} \cdot M_{d}$ is a diagonal form $\Delta_{v}$. Then it is a standard argument (see for example [2, Lemma 2.2]) that the groups $\mathrm{SU}\left(Q_{v}, \mathcal{O}_{k}, \tau_{d}\right)$ and $\mathrm{SU}\left(\Delta_{v}, \mathcal{O}_{k}, \tau_{d}\right)$ are commensurable, so at possibly the expense of passing to a further subgroup of finite index, we obtain a representation

$$
r_{d, u}: H_{d, u} \longrightarrow \mathrm{SU}\left(\Delta_{v}, \mathcal{O}_{k}, \tau_{d}\right)
$$

as required by Theorem 1.1 .

\section{Projective considerations: $r_{d}$ is faithful}

This section contains the proof that one can find many representations $r_{d}$ which are faithful. It is basically geometric in nature and relies upon the fact that these representations are associated to convex real projective structures on the figure-eight knot. 
Throughout we denote the complement of the figure-eight knot in $\mathbf{S}^{3}$ by $M$ and denote its fundamental group by $\Gamma$. We begin with some considerations of a fairly general nature. We recall that a group is said to be nonradical if there is no infinite normal nilpotent subgroup. The figure-eight knot group (and indeed any finite-volume hyperbolic manifold group) is nonradical. The following is a classical theorem of Zassenhaus.

Theorem 3.1 (Kapovich [13, Theorem 8.4]) Suppose that $G$ is a finitely generated nonradical group and $\mathcal{L}$ any linear Lie group and that $\left\{\sigma_{n}\right\}$ is a convergent sequence of discrete, faithful representations of $G$ into $\mathcal{L}$, say $\sigma_{n} \rightarrow \sigma_{\infty}$. Then $\sigma_{\infty}$ is discrete and faithful.

We will also need the following:

Theorem 3.2 (Goldman and Choi [9]) Let $G$ be a finitely generated nonradical group. Suppose that $\Omega_{n}$ is a sequence of properly convex open domains in $\mathbf{R P}^{d}$ and $\sigma_{n}: G \rightarrow \operatorname{SL}(d+1, \mathbf{R})$ a convergent sequence of discrete faithful representations for which $\sigma_{n}(G)<\operatorname{Aut}\left(\Omega_{n}\right)$. Denote the limit representation by $\sigma_{\infty}$. Then if $\sigma_{\infty}$ is irreducible, it preserves some properly convex open subset of $\mathbf{R P}^{d}$.

We define a subset of the representation variety $\Omega\left(G ; \mathbf{R P}^{d}\right)<\operatorname{Hom}(G, \operatorname{SL}(d+1, \mathbf{R}))$ to be the set of representations $\sigma: G \rightarrow \operatorname{SL}(d+1, \mathbf{R})$ satisfying:

- $\sigma$ is discrete and faithful.

- There is a properly convex, open domain $\Omega_{\sigma} \subset \mathbf{R P}^{d}$ for which $\sigma(G)<\operatorname{Aut}\left(\Omega_{\sigma}\right)$.

In the case that $G$ is the fundamental group of eg a hyperbolic $d$-manifold, the set $\Omega\left(G ; \mathbf{R P}^{d}\right)$ is nonempty, since it contains the representation corresponding to the complete structure $\rho_{1}=\phi_{1 / 2}$.

Theorem 3.3 The path component of the set $\mathcal{G}=\left\{v \mid \rho_{v} \in \Omega\left(\Gamma ; \mathbf{R P}^{3}\right)\right\}$ which contains 1 is open and closed.

In particular, once Theorem 3.3 is proven, we have that $\mathcal{G}$ is some interval, and therefore $\mathcal{G}=(0, \infty)$ since the only place the representations $\rho_{v}$ fail to be defined is at $v=0$. This implies that any specialization of $v$ in $(0, \infty)$ is discrete and faithful, so that taken in conjunction with Theorem 2.6, we will have proved all of Theorem 1.1, barring the fact the image is Zariski dense. 
Proof of Theorem 3.3 The fact that the set is closed follows from a concatenation of results proved above: Section 3 implies that the endpoint of a path of discrete faithful representations is discrete and faithful, then Theorem 3.2 implies the resulting representation is the holonomy of a properly convex structure, since we proved in Theorem 2.1 that the limit representation is irreducible.

Openness is a good deal more subtle. By applying a theorem of Cooper and Long [10, Theorem 0.4] we find that a small deformation of the holonomy of the complete hyperbolic structure of $M$ whose restriction to some (hence any) peripheral subgroup has a common fixed point in $\mathbf{R} \mathbf{P}^{3}$ will itself be the holonomy of a projective structure on $M$. Furthermore, the cusp of this projective structure is foliated by projective rays with a common endpoint.

A priori, this projective structure need not be properly convex. However the main result of [11] shows that if the cusp of the resulting projective structure satisfies slightly stronger hypotheses then the deformed projective structure will be properly convex. Roughly speaking, the additional hypothesis is that the cusp of the deformed projective structure must admit a second foliation by "strictly convex" hypersurfaces that is transverse to the previously mentioned foliation by projective rays.

In [4] it is shown that for all $t \in(0, \infty)$ the representation $\rho_{t}$ satisfy the hypotheses of the previously mentioned theorems. Since the two families of representations are conjugate this implies that for all $u \in(0, \infty), \rho_{u}$ also satisfy the same hypotheses. As a result we find that for $u$ sufficiently close to 1 that $\rho_{u}$ is the holonomy of a properly convex projective structure on $M$. The holonomy of such a structure is necessarily discrete and faithful and so for $u$ sufficiently close to 1 we find that $\rho_{u} \in \Omega\left(\Gamma ; \mathbf{R P}^{3}\right)$. We thus conclude that $\mathcal{G}$ is open.

\section{Zariski denseness}

In this section we analyze the Zariski closure of the groups $\phi_{t}(\Gamma)$ (which we denote $G_{t}$ ) proving in particular that away from the complete representation, this Zariski closure is all of $\operatorname{SL}(n, \mathbf{R})$. We adopt a largely geometric point of view; other approaches are possible, see the remarks at the conclusion of this section.

We begin by with some background and results which can be found in Benoist [8]. A comprehensive summary of the necessary background in algebraic groups, notions of proximality, and Zariski closures can be found in [16; 17]. We begin by defining proximality in the context of groups, group actions, and representations. In all cases proximality is related to the existence of unique attracting fixed points. 
Let $G$ be a subgroup of $\operatorname{SL}(n, \mathbf{R})$ and let $g$ be an element of $G$. We say that $g$ is proximal if it has a unique eigenvalue of largest modulus. In this case it is easy to see that this eigenvalue is real. If in addition this eigenvalue of largest modulus is positive then we say that $g$ is positive proximal. A group is called proximal if it contains a proximal element and positive proximal if every proximal element is positive proximal. A group action of $G$ on $\mathbf{R} \mathbf{P}^{n-1}$ is proximal if for any pair of points $x, y \in \mathbf{R} \mathbf{P}^{n-1}$ there is a sequence $\left\{g_{m}\right\}$ of elements in $G$ such that

$$
\lim _{m \rightarrow \infty} g_{m} \cdot x=\lim _{m \rightarrow \infty} g_{m} \cdot y .
$$

If $G$ is a connected semisimple Lie group then a representation $\rho: G \rightarrow \operatorname{SL}(n, \mathbf{R})$ is proximal if the weight space corresponding to highest restricted weight is 1-dimensional. More specifically, if we let $G=K A N$ be an Iwasawa decomposition of $G$, where $K$ is a maximal compact subgroup, $A$ is a maximal abelian subgroup, and $N$ is a maximal nilpotent subgroup, then the set

$$
\left\{x \in \mathbf{R}^{n} \mid n(x)=x \text { for all } n \in N\right\}
$$

is a line.

We now discuss some relations between these notions. We say that a group $\Gamma \subset \operatorname{SL}(n, \mathbf{R})$ is strongly irreducible if every finite-index subgroup of $\Gamma$ is irreducible. The following two theorems relate the various notions of proximality.

Theorem 4.1 [12, Theorem 2.9] Let $G$ be a subgroup of $\operatorname{SL}(n, \mathbf{R})$. The following are equivalent:

(1) $G$ is strongly irreducible and proximal.

(2) $G$ is irreducible and its action on $\mathbf{R P}^{n-1}$ is proximal.

Theorem 4.2 [1, Theorem 6.3] Let $G$ be a semisimple Lie group with finite center and let $\rho: G \rightarrow \operatorname{SL}(n, \mathbf{R})$ be an irreducible representation. The following are equivalent:

(1) $\rho$ is proximal.

(2) $\rho(G)$ is proximal.

Given a strongly irreducible and proximal subgroup $G \subset \operatorname{SL}(n, \mathbf{R})$ we can define the limit set of $G$, which we denote by $\Lambda_{G}$ as the closure of the set of attractive fixed points in $\mathbf{R} \mathbf{P}^{n-1}$ of the proximal elements of $G$. In [12, Theorem 2.3] it is shown that the action of $G$ on $\Lambda_{G}$ is minimal (ie any nonempty closed $G$-invariant subset of $\mathbf{R} \mathbf{P}^{n-1}$ contains $\Lambda_{G}$ ). 
When $G$ is a Zariski closed semisimple subgroup of $\operatorname{SL}(n, \mathbf{R})$ we can describe $\Lambda_{G}$ more explicitly. Since $G$ is proximal, $\Lambda_{G}$ is nonempty and so let $x \in \Lambda_{G}$. Consider the orbit $G \cdot x$ of $x$. First, observe that $G \cdot x \subset \Lambda_{G}$ by $G$-invariance. Furthermore, since $G \cdot x$ is the orbit of an algebraic group acting algebraically on a variety we have that $G \cdot x$ is a smooth subvariety of $\mathbf{R} \mathbf{P}^{n-1}$. If we let $H$ be the Zariski closure of $G \cdot x$ then we see that $G \cdot x$ is open in $H$ and so $H \backslash(G \cdot x)$ is a Zariski closed subset of $\mathbf{R P}^{n-1}$. Since Zariski closed sets are closed in the standard topology, minimality implies that $H \backslash(G \cdot x)$ is either empty or $\Lambda_{G}$. However, $x \notin H \backslash(G \cdot x)$ and so $H \backslash(G \cdot x)$ is empty. As a result we see that $G \cdot x$ is a nonempty closed $G$-invariant subset and thus $G \cdot x=\Lambda_{G}$. By minimality of the action on the limit set we also find that $\Lambda_{G}$ is the unique closed orbit for the action of $G$ on $\mathbf{R P}^{n-1}$.

We can say even more. Let $G=K A N$ be an Iwasawa decomposition and let $x_{N}$ be the (unique) point in $\mathbf{R P}^{n-1}$ corresponding to a highest-weight vector. By our choice of $x_{N}$ we see that the groups $A$ and $N$ both fix $x_{N}$ and so $G \cdot x_{N}=K \cdot x_{N}$. Thus the orbit of $x_{N}$ is closed and so $G \cdot x_{N}=\Lambda_{G}$ (ie the limit set of $G$ is the orbit of a compact group.)

We can now identify the Zariski closure of $\phi_{t}(\Gamma)$, which we denote $G_{t}$. The representation $\phi_{1 / 2}$ corresponds to the holonomy of the complete finite-volume hyperbolic structure on the figure-eight knot and so by the Borel density theorem we have $G_{1 / 2}=\operatorname{SO}(3,1)$. The main goal of this section is to show that when $t \neq \frac{1}{2}$ that $G_{t}=\operatorname{SL}(4, \mathbf{R})$. The proof is based on the following heuristic:

A Lie subgroup of $\mathrm{SL}(4, \mathbf{R})$ with a large orbit in $\mathbf{R} \mathbf{P}^{3}$ must be large.

Theorem 4.3 If $t \neq \frac{1}{2}$ then $G_{t}=\operatorname{SL}(4, \mathbf{R})$. In particular, for $t \neq \frac{1}{2}, \phi_{t}(\Gamma)$ is Zariski dense.

Proof Let $t \neq \frac{1}{2}$. We have previously seen that $\rho_{t}$ is an absolutely irreducible representation whose image preserves a properly convex open subset of $\mathbf{R P}^{3}$. We first show that $\rho_{t}(\Gamma)$ is strongly irreducible. Suppose for contradiction that $\rho_{t}(\Gamma)$ is not strongly irreducible. By work of Benoist [8, Lemma 2.9] we can find a finite-index subgroup of $\Gamma$ that splits as a nontrivial direct sum. However, any finite-index subgroup of $\Gamma$ is the fundamental group of a finite-volume hyperbolic 3-manifold and such groups never admit nontrivial direct sum decompositions.

Let $G_{t}^{0}$ be the connected component of $G_{t}$ containing the identity. We claim that $G_{t}^{0}$ is semisimple. To see this observe that by [12, Lemma 2.6] we find that $G_{t}^{0}$ acts irreducibly on $\mathbf{R}^{4}$. As a result the action of $G_{t}^{0}$ turns $\mathbf{R}^{4}$ into a simple $\mathbf{R}\left[G_{t}^{0}\right]$ module. Let $R_{t}$ be the unipotent radical of $G_{t}^{0}$. Since $R_{t}$ is unipotent and solvable 
the Lie-Kolchin theorem implies that there is a nontrivial $\mathbf{C}\left[R_{t}\right]-$ submodule, $E_{\mathbf{C}}$, of simultaneous 1-eigenvectors of $R_{t}$ in $\mathbf{C}^{4}$. A simple computation shows that $E_{\mathbf{C}}$ is conjugation invariant and so there is a nontrivial $\mathbf{R}\left[R_{t}\right]$-submodule, $E_{\mathbf{R}} \subset \mathbf{R}^{4}$, of simultaneous 1-eigenvectors of $R_{t}$ whose complexification is $E_{\mathbf{C}}$. However, since $R_{t}$ is normal in $G_{t}^{0}$ we see that $E_{\mathbf{R}}$ is a nontrivial $\mathbf{R}\left[G_{t}^{0}\right]$-submodule. However, simplicity implies that this submodule must be all of $\mathbf{R}^{4}$. Therefore $R_{t}$ acts trivially on $\mathbf{R}^{4}$ and is thus trivial. We conclude that $G_{t}^{0}$ is reductive. Furthermore, since $G_{t}^{0}$ is proximal and irreducible we see that it has trivial center and is thus semisimple.

The group $\phi_{t}(\Gamma)$ is proximal and so Theorem 4.2 implies that the representation induced by the inclusion of $G_{t}^{0}$ into $\operatorname{SL}(4, \mathbf{R})$ is also proximal.

Next we show that $\Lambda_{G_{t}^{0}}$ contains a codimension-1 submanifold. Let $\Gamma_{p}$ be a peripheral subgroup of $\Gamma$. By work of $\left[4\right.$, Section 6] it is possible to conjugate so that $\phi_{t}\left(\Gamma_{p}\right)$ to a lattice in the 2-dimensional abelian Lie group $H$ of matrices of the form

$$
\left(\begin{array}{cccc}
1 & 0 & s & s^{2} / 2-t \\
0 & e^{t} & 0 & 0 \\
0 & 0 & 1 & s \\
0 & 0 & 0 & 1
\end{array}\right) .
$$

Thus we see that the Zariski closure of $\phi_{t}\left(\Gamma_{p}\right)$ (and hence $G_{t}$ ) contains $H$. A generic orbit of $H$ can be written in homogenous coordinates as

$$
\left\{\left[-\log (|x|)+y^{2} / 2+c: \epsilon x: y: 1\right] \mid x>0\right\},
$$

where $\epsilon \in\{ \pm 1\}$ and $c \in \mathbf{R}$ (see Figure 1). Since $G_{t}^{0}$ is irreducible we see that $\Lambda_{G_{t}^{0}}$ contains a point, $z$ of one of these orbits and so $H \cdot z \subset G \cdot z \subset \Lambda_{G_{t}^{0}}$. Furthermore, since $\Lambda_{G_{t}^{0}}$ is closed we see that it contains the closure of this orbit, which is the boundary of a properly convex domain $\Omega \subset \mathbf{R P}^{3}$.

There are now two cases to consider: either $\Lambda_{G_{t}^{0}}$ has codimension 0 and is thus equal to $\mathbf{R} \mathbf{P}^{3}$ or $\Lambda_{G_{t}^{0}}$ is a codimension-1 submanifold of $\mathbf{R P}^{3}$ and is thus equal to $\partial \Omega$. In the first case work of Benoist [8, Lemma 3.9 and Corollary 3.5] shows that $G_{t}^{0}=\operatorname{SL}(4, \mathbf{R})$, in which case we are done.

We now rule out the second case. In this case we see that there is a point $w \in \mathbf{R P}^{3}$ such that $\Lambda_{G_{t}^{0}}=K \cdot w=\partial \Omega$, where $K$ is a maximal compact subgroup of $G_{t}^{0}$. As a result we see that (up to a subgroup of index 2) $K$ preserves $\Omega$. Since $K$ is compact we see that there is a point $x_{0} \in \Omega$ such that both $x_{0}$ and its dual point $x_{0} \in \Omega^{*}$ are both fixed by $K .^{1}$ The hyperplane dual to $x_{0}^{*}$ provides us an affine patch containing

\footnotetext{
${ }^{1}$ See [17, Section 9] for a definition of dual point of properly convex domains.
} 


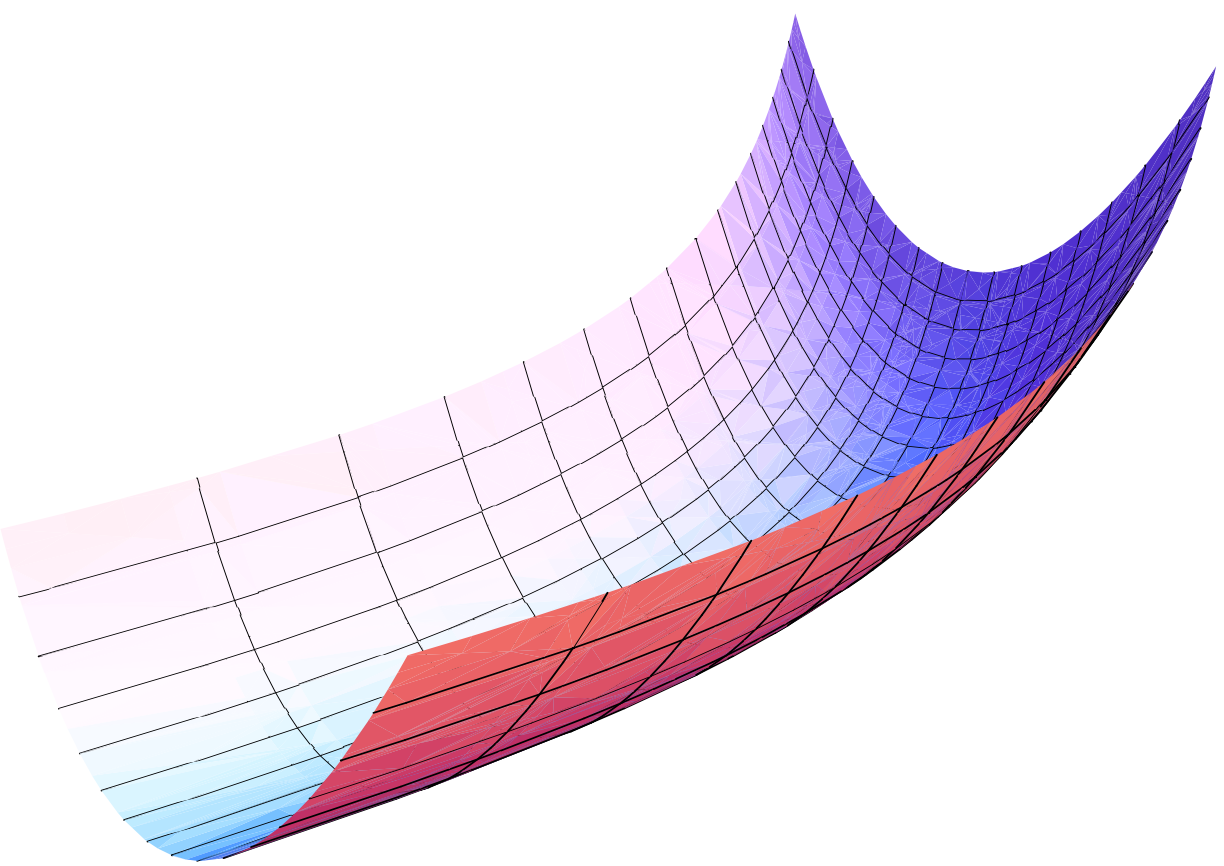

Figure 1: A generic orbit of $H$

$\Omega$ and for which $x_{0}$ is the center of mass of $\Omega$. In these affine coordinates $K$ acts by affine transformations fixing $x_{0}$.

If we let $\mathcal{E}$ be a John ellipsoid ${ }^{2}$ for $\Omega$ centered at $x_{0}$ in this affine patch, then we see that $K$ preserves $\mathcal{E}$. Since $\mathcal{E}$ is has maximal volume $\partial \mathcal{E}$ has nonempty intersection with $\partial \Omega$. Since $K$ acts transitively on $\partial \Omega$ we see that $\partial \Omega \subset \partial \mathcal{E}$.

For dimensional reasons we see that $\partial \Omega$ has nonempty interior in $\partial \mathcal{E}$. Since $\partial \Omega$ is a $K$-orbit we see that it is an open subset of $\partial \mathcal{E}$. We conclude that $\partial \Omega=\partial \mathcal{E}$ and thus $\Omega$ is an ellipsoid. Since $\phi_{t}(\Gamma)$ preserves this ellipsoid we see that it is conjugate to a subgroup of $\mathrm{SO}(3,1)$. This is a contradiction since, for example the image of the longitude has an eigenvalue whose inverse is not also an eigenvalue.

Remark Given the explicit nature of the subgroups in this paper there are alternative, more algebraic ways of proving Zariski denseness. For example, it suffices to show that the adjoint action of $\rho_{t}(\Gamma)$ on $\mathfrak{s l}_{4}$ is irreducible (a Mathematica notebook with irreducibility calculations for our groups can be found at [5]). The anonymous referee

\footnotetext{
${ }^{2}$ A John ellipsoid for a convex subset $\Omega$ of affine space is an ellipsoid of maximal Euclidean volume contained in $\Omega$ with the same center of mass.
} 
also pointed out other techniques that could be employed to show that such explicit subgroups are Zariski dense (see [21] and [19, Theorem 9.10]). We chose to use the above proof because of its geometric nature which highlights the relationship between convex projective structures and the Zariski closure of their holonomy representations. We plan to pursue this relationship in more detail in future work.

\section{Appendix: The matrices}

The discrete faithful representation corresponding to the hyperbolic structure occurs at $v=1$ :

$$
\begin{gathered}
\rho_{v}(x)=\left(\begin{array}{rrrr}
\frac{3}{2} & \frac{1}{2} & \frac{1}{2}(1+1 / v) & \frac{1}{\sqrt{12}}(1-1 / v) \\
-\frac{1}{2} & \frac{1}{2} & -\frac{1}{2}(1+1 / v) & -\frac{1}{\sqrt{12}}(1-1 / v) \\
1 & 1 & 1 & 0 \\
0 & 0 & 0 & 1
\end{array}\right), \\
\rho_{v}(y)=\left(\begin{array}{cccc}
v+\frac{1}{2} & -v+\frac{1}{2} & \frac{1}{2} & \frac{1}{\sqrt{12}}(7-4 v) \\
\frac{1}{2} & \frac{1}{2} & \frac{1}{2} & \frac{1}{\sqrt{12}}(4 / v-1) \\
\frac{1}{2} v & -\frac{1}{2} v & 1 & \frac{1}{\sqrt{3}}(1-v) \\
\frac{\sqrt{3}}{2} v & -\frac{\sqrt{3}}{2} v & 0 & 2-v
\end{array}\right) .
\end{gathered}
$$

The discrete faithful representation corresponding to the hyperbolic structure occurs at $t=\frac{1}{2}$ :

$$
\phi_{t}(x)=\left(\begin{array}{llcc}
1 & 0 & 1 & t-1 \\
0 & 1 & 1 & t \\
0 & 0 & 1 & t+\frac{1}{2} \\
0 & 0 & 0 & 1
\end{array}\right), \quad \phi_{t}(y)=\left(\begin{array}{cccc}
1 & 0 & 0 & 0 \\
2+1 / t & 1 & 0 & 0 \\
2 & 1 & 1 & 0 \\
1 & 1 & 0 & 1
\end{array}\right) \text {. }
$$

The representations become conjugate for $v=2 t$.

\section{References}

[1] H Abels, G A Margulis, G A Soŭfer, Semigroups containing proximal linear maps, Israel J. Math. 91 (1995) 1-30 MR1348303

[2] I Agol, D D Long, A W Reid, The Bianchi groups are separable on geometrically finite subgroups, Ann. of Math. 153 (2001) 599-621 MR1836283

[3] S A Ballas, Deformations of noncompact projective manifolds, Algebr. Geom. Topol. 14 (2014) 2595-2625 
[4] S A Ballas, Finite volume properly convex deformations of the figure-eight knot, Geom. Dedicata 178 (2015) 49-73 MR3397481

[5] S Ballas, D D Long, Computation: Irreducibility Mathematica notebook. Available at http://tinyurl.com/ogx2vn9

[6] S Ballas, D D Long, Computation: Representation traces Mathematica notebook. Available at http://tinyurl.com/qxs94cq

[7] H Bass, Groups of integral representation type, Pacific J. Math. 86 (1980) 15-51 MR586867

[8] Y Benoist, Automorphismes des cônes convexes, Invent. Math. 141 (2000) 149-193 MR1767272

[9] S Choi, W M Goldman, Convex real projective structures on closed surfaces are closed, Proc. Amer. Math. Soc. 118 (1993) 657-661 MR1145415

[10] D Cooper, D D Long, A generalization of the Epstein-Penner construction to projective manifolds, Proc. Amer. Math. Soc. 143 (2015) 4561-4569 MR3373953

[11] D Cooper, D D Long, S Tillmann, Deforming convex projective manifolds in preparation

[12] I Y Goldsheid, Y Guivarc'h, Zariski closure and the dimension of the Gaussian law of the product of random matrices, I, Probab. Theory Related Fields 105 (1996) 109-142 MR1389734

[13] M Kapovich, Hyperbolic manifolds and discrete groups, Progress in Mathematics 183, Birkhäuser, Boston, MA (2001) MR1792613

[14] S Lang, Algebra, 3rd edition, Graduate Texts in Mathematics 211, Springer, New York (2002) MR1878556

[15] D D Long, A W Reid, M Thistlethwaite, Zariski dense surface subgroups in $\operatorname{SL}(3, \mathbf{Z})$, Geom. Topol. 15 (2011) 1-9 MR2764111

[16] L Marquis, Coxeter group in Hilbert geometry arXiv: 1408.3933

[17] L Marquis, Around groups in Hilbert geometry, from: "Handbook of Hilbert geometry", (A Papadopoulos, M Troyanov, editors), IRMA Lect. Math. Theor. Phys. 22, Eur. Math. Soc., Zürich (2014) 207-261 MR3329882

[18] D W Morris, Introduction to arithmetic groups (2008) arXiv:math/0106063v3

[19] G Prasad, A S Rapinchuk, Generic elements in Zariski-dense subgroups and isospectral locally symmetric spaces, from: "Thin groups and superstrong approximation", (E Breuillard, H Oh, editors), MSRI Publ. 61, Cambridge Univ. Press (2014) 211-252 MR3220892

[20] I Reiner, Maximal orders, London Math. Soc. Monographs 28, Oxford University Press (2003) MR1972204 
[21] I Rivin, Large Galois groups with applications to Zariski density (2015) arXiv: 1312.3009v4

[22] P Sarnak, Notes on thin groups (2012) Available at http://www.msri.org/ attachments/workshops/652_Sarnak-notes.pdf

Department of Mathematics, University of California Santa Barbara Santa Barbara, CA 93106-3080, USA

sballas@math.ucsb.edu, long@math.ucsb.edu

http://www.math.ucsb.edu/ sballas, http://www.math.ucsb.edu/ long

Received: 29 October $2014 \quad$ Revised: 24 December 2014 